\title{
Network Robot Systems
}

\author{
Alberto Sanfeliu ${ }^{1}$, Norihiro Hagita ${ }^{2}$ and Alessandro Saffiotti ${ }^{3}$ \\ Guest Editors of the Special Issue on NRS \\ ${ }^{1}$ Institut de Robòtica I Informàtica Industrial (UPC-CSIC), Universitat Politècnica de Catalunya \\ Llorens I Artiga, 4-6, 08028 Barcelona, Spain \{sanfeliu@iri.upc.edu\} \\ ${ }^{2}$ ATR Intelligent Robotics and Communication Labs \& ATR Media Information Science Labs \\ 2-2-2 Hikaridai, "Keihanna Science City", Kyoto 619-0288 Japan \{hagita@atr.jp\} \\ ${ }^{3}$ AASS Mobile Robotics Lab, Dept of Technology, Orebro University , \\ S-70182 Orebro, Sweden \{asaffio@aass.oru.se
}

\begin{abstract}
This article introduces the definition of Network Robot Systems (NRS) as is understood in Europe, USA and Japan. Moreover, it describes some of the NRS projects in Europe and Japan and presents a summary of the papers of this Special Issue.
\end{abstract}

Keywords: network robot, ubiquitous robotics, cooperative robotics, mobile robots, wireless sensor network, telerobotics

\section{1) Definition of Network Robot Systems}

The technological development of the last decade in robots, computing and communications has led to envisage the design of robotic and automation systems consisting of networked vehicles, sensors, actuators and communication devices. These developments enable researchers and engineers to design new robotic systems that can interact with human beings and other robots in a cooperative way. This new technology has being denominated "Network Robot Systems" (NRS) and includes the following elements:

- $\quad$ Physical embodiment: Any NRS has to have at least a physical robot which incorporates hardware and software capabilities

- Autonomous capabilities: A physical robot must have autonomous capabilities to be considered as a basic element of a NRS.

- $\quad$ Network based cooperation: The robots, environment sensors and humans must communicate and cooperate through a network.

- Environment sensors and actuators: Besides the sensors of the robots, the environment must include other sensors, such as vision cameras and laser range finders, and other actuators, such as speakers and flickers

- Human-robot interaction: In order to consider a system as NRS, the system must have a humanrobot related activity.

The name Networked Robots (NR) was created in May 2004 within the IEEE RAS Technical Committee, as a consequence of the preliminary work on Internet-based tele-operated robots initiated in 2001, and its expansion to reflect a broader set of problems and applications. The IEEE Society of Robotics and Automation's Technical Committee on Networked Robots made the following definition of Networked Robots:

“A 'networked robot' is a robotic device connected to a communications network such as the Internet or LAN. The network could be wired or wireless, and based on any of a variety of protocols such as TCP, UDP, or 802.11. Many new applications are now being developed ranging from automation to exploration. There are two subclasses of Networked Robots: (1) Tele-operated, where human supervisors send commands and receive feedback via the network. Such systems support research, education, and public awareness by making valuable resources accessible to broad audiences; (2) Autonomous, where robots and sensors exchange data via the network. In such systems, the sensor network extends the effective sensing range of the robots, allowing them to communicate with each other over long distances to coordinate their activity. The robots in turn can deploy, repair, and maintain the sensor network to increase its longevity, and utility. A broad challenge is to develop a science base that couples communication to control to enable such new capabilities.” 
This concept was expanded by a Japanese study group on network robot organized by Ministry of Internal Affairs and Communications of Japan, which included three types of network robots: visible, virtual, and unconscious - where the latter term indicates that the users are not aware of the presence of the robot. The visible robot is literally a robot that can be seen and it can take various forms of body such as humanoid, pet, stuffed animal and so on. The virtual type of robot acts in a cyber space and makes use of information available on Internet. The avatar agent that appears on a mobile phone or personal computer is a typical example. The unconscious type robot means a camera or a sensor with CPU, which is embedded in roads, town, room or equipment, or is hidden in clothes or accessories.

A simpler definition was proposed by a European study group "Research Atelier on Network Robot Systems” inside of EURON II (www.euron.org). The definition is the following:

"A Network Robot System is a group of artificial autonomous systems that are mobile and that make important use of wireless communications among them or with the environment and living systems in order to fulfil their tasks".

Network Robot Systems (NRS) call for the integration of several fields: robotics, perception (sensor systems), ubiquitous computing, artificial intelligence, and network communications. Some of the key issues that must be addressed in the design of Network Robot Systems are cooperative localization and navigation, cooperative environment perception, cooperative map building, task allocation, cooperative task execution, human-robot interaction, network teleoperation, and communications.

The topic Network Robot Systems transcends “conventional” robotics, in the sense that there exists, for these type of distributed heterogeneous systems, an interrelation among a community of robots, environment sensors and humans. Applications include network robot teams (for example to play soccer), human-robot networked teams (for example a community of robots that assist people), robots networked with the environment (for example for tasks on urban settings or in space applications) or geminoid robots (a replication of a human with own autonomy and being partially tele-operated through the network).

\section{2) Some major collaborative efforts in NRS}

The papers in this Special Issue give a snapshot of the current state of the art in dealing with the technical issues involved in NRS. In order to provide the reader with an overall perspective on this new field, we briefly describe three major on-going projects in NRS.

\section{1) Ubiquitous Robotics Network System for Urban Settings (URUS)}

The general objective of the URUS project (http://urus.upc.es) is the development of new ways of cooperation between network robots and human beings and/or the environment in urban areas, in order to achieve efficiently tasks that in the other way can be very complex, time consuming or too costly. The focus of the project is in urban pedestrian areas, an important topic in Europe where there exists a growing interest in reducing the number of cars in the streets and improving the quality of life. Network robots can be an important instrument to address these issues in the cities.

The URUS project [1] is focused on designing and developing a network of robots that in a cooperative way interact with human beings and the environment for tasks of guidance and assistance, transportation of goods, and surveillance in urban areas. The networked robot architecture integrates cooperating urban robots, intelligent sensors (video cameras, acoustic sensors, etc.), intelligent devices (PDA, mobile telephones, etc.) and communications. The main scientific and technological challenges of the project are: navigation and motion coordination among robots; cooperative environment perception; cooperative map building and updating; task negotiation within cooperative systems; human robot interaction; and wireless communication strategies between users (mobile phones, PDAs), the environment (cameras, acoustic sensors, etc.), and the robots.

The architecture of the project is shown in Fig.1 and integrates robots, environment sensors and humans, all connected through WiFi technology. 


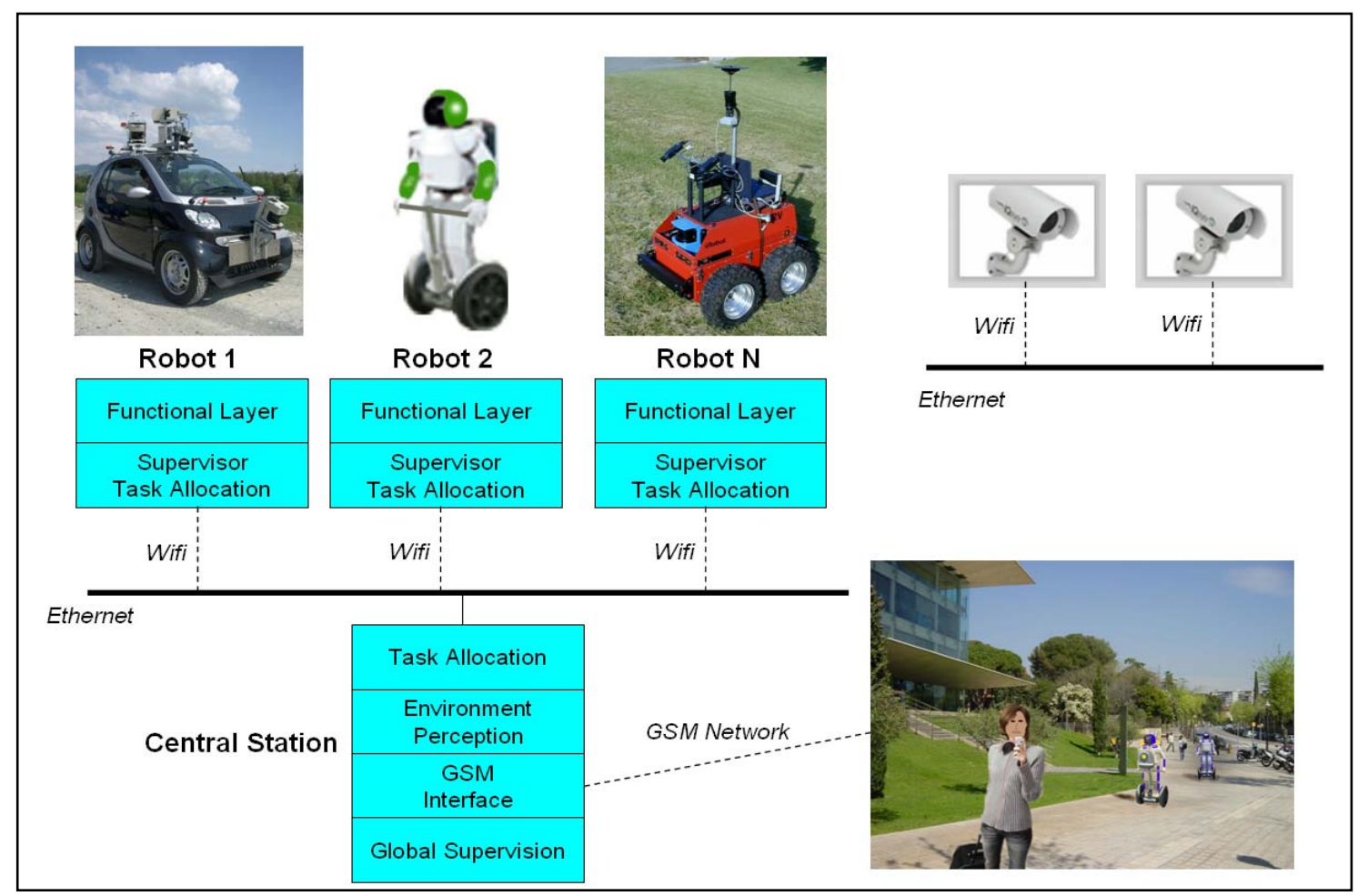

Fig.1 URUS architecture

The URUS project will be tested in an Urban Setting, $\left(10.000 \mathrm{~m}^{2}\right.$ in the North Campus of the Technical University of Catalonia in Barcelona city, see Fig.2) and three experiments are planned: (1) transportation of persons; (2) guidance of people; and (3) evacuation of people.

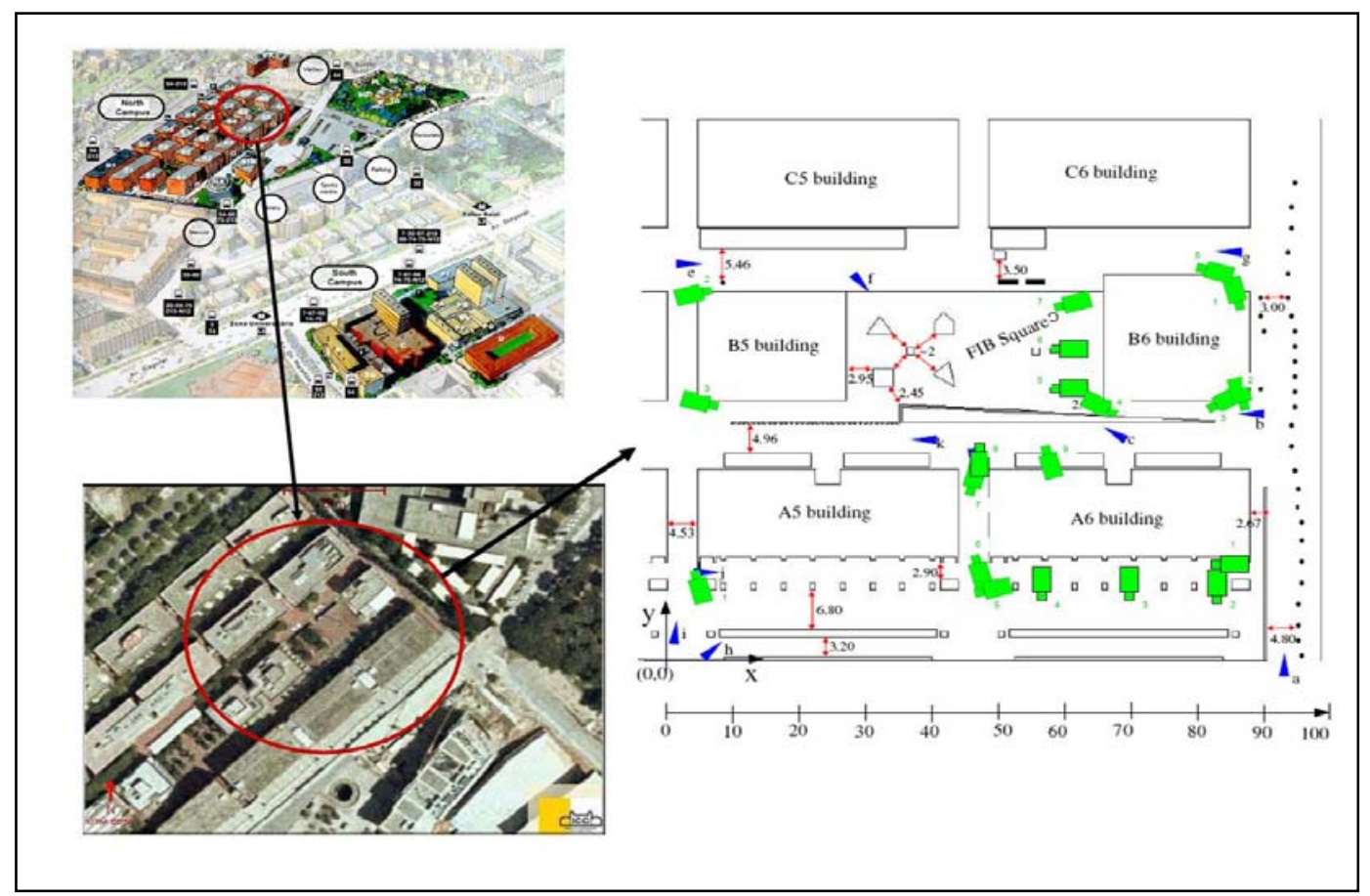

Fig.2 Permanent test site in North Campus of Technical University of Catalonia (Barcelona)

The URUS project involves 11 European partners of 6 different countries and the coordinator is the Technical University of Catalonia. 


\section{2) Japan NRS project}

Japan's NRS project started in 2004. Its objective is to enable user-friendly interaction between humans and networked environments. Visible robots, such as humanoids and pet robots, perform the role of providing information to people, while observation and recognition of people are mainly handled by the sensors embedded in the environment. There are four main aspects to this project: communication among robots, the networking platform, ubiquitous sensing, and human-robot interaction based on NRS.

The Japan NRS project has two unique features: First, they have a unique focus on human-robot interaction. Their robots do not perform physical tasks, but instead engage in communication tasks with users. Second, there is a strong emphasis on bringing robots out of the laboratory and into real field settings. One notable example of such a field study is a museum guide robot network using ubiquitous sensors [2], where they demonstrated live examples of human-robot interaction enabled by NRS.

The Japan NRS project consists of four major Japanese companies: NTT, a telecommunication company; Toshiba, a home appliance company; Mitsubishi Heavy Industries, a heavy industry company whose products include industrial robots; and ATR, a research-oriented company for telecommunication and social robotics.

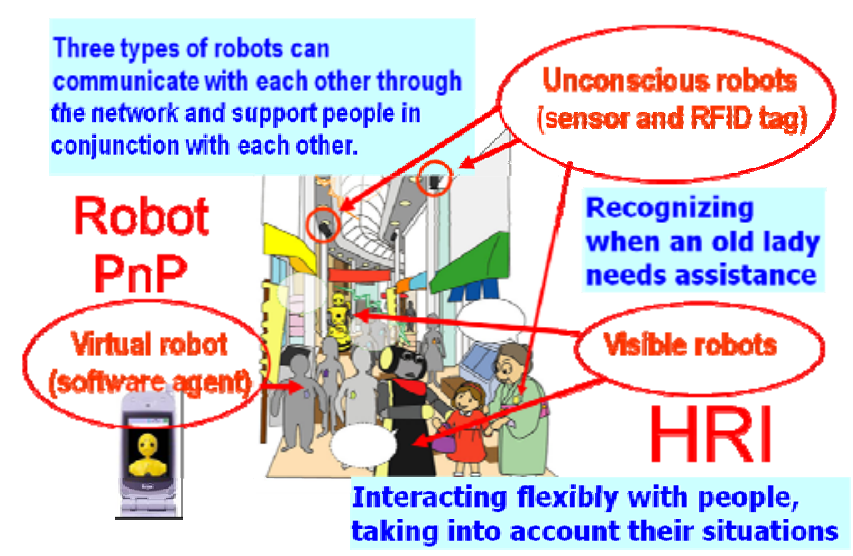

Fig.3 Concept of Japan NRS

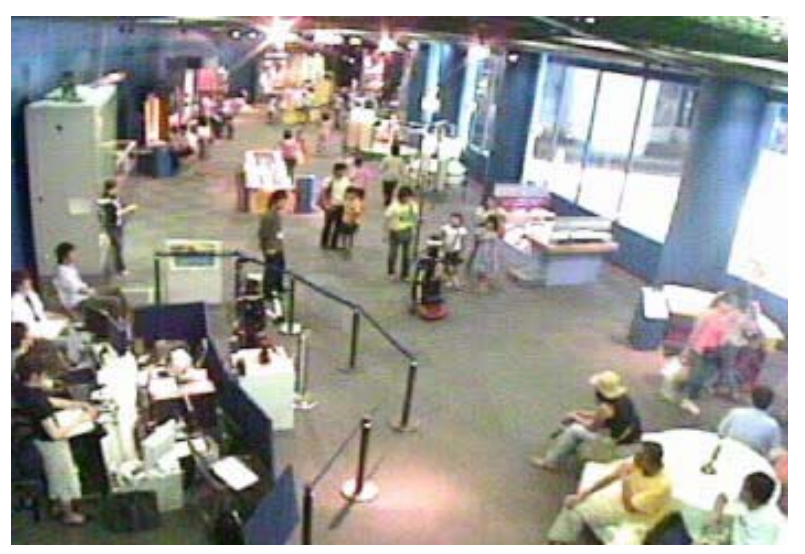

Fig.4 Field trial of Japan NRS at a science museum

\section{3) PEIS Ecology project}

The concept of Ecology of Physically Embedded Intelligent Systems, or PEIS Ecology, combines insights from the fields of ubiquitous robotics and ambient intelligence to provide a new solution to building intelligent robotic systems in the service of people [3]. The PEIS Ecology approach takes an ecological viewpoint in which robots, humans and devices in the environments are seen as parts of the same ecosystem, engaged in a symbiotic relationship toward the achievement of common goals or equilibria. Figure 5 illustrates the concept of PEIS Ecology. Essential to this concept is the fact that the same 
components can be automatically re-configured to perform different tasks and to adapt to different contextual conditions.

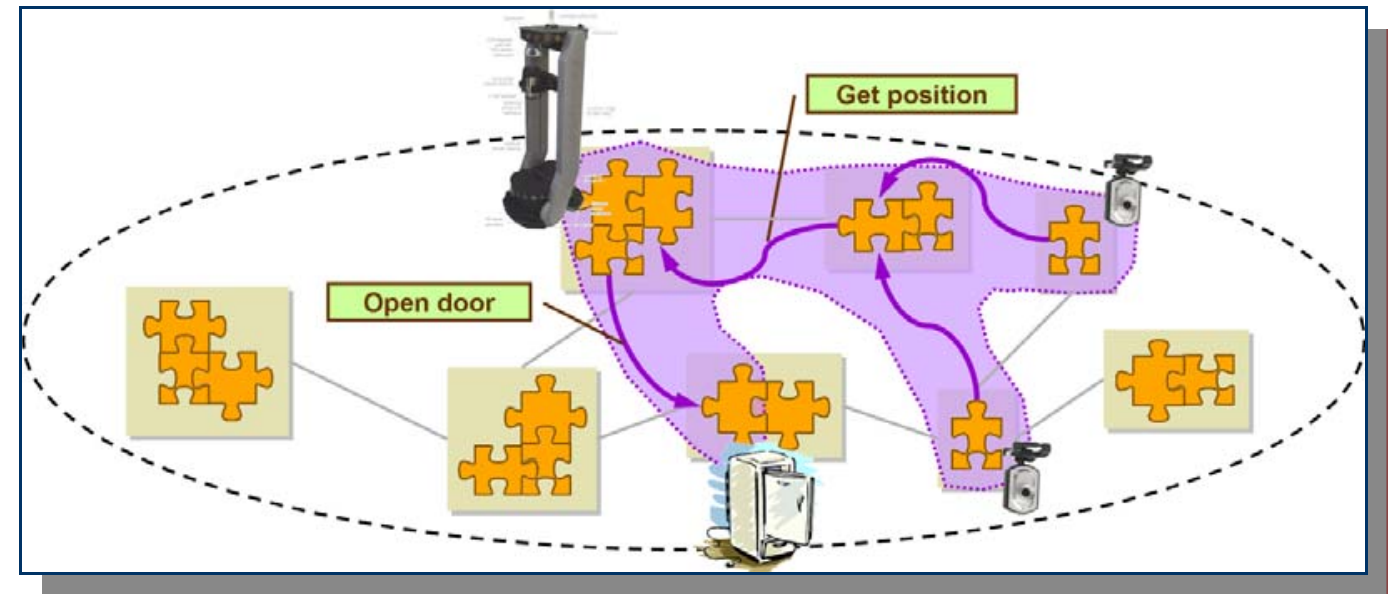

Fig. 5: A simple PEIS Ecology in which a robot, a refrigerator, and two cameras co-operate in the performance of a task.
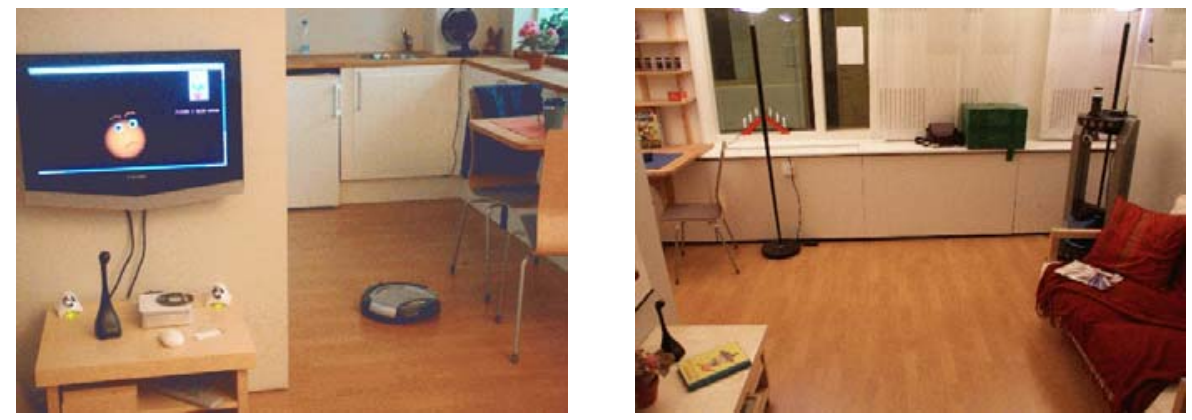

Fig. 6: Two views of the PEIS Ecology test environment.

The PEIS Ecology approach has been developed in the framework of a cooperative project between the University of Orebro, Sweden, and ETRI (Electronic and Telecommunication Research Institute), Korea. This project is distinct in its emphasis on the fundamental scientific principles that underlie the design and operation of network robot systems, including issues such as: middleware for highly heterogeneous distributed systems, self-configuration and dynamic re-configuration, cooperative perception, and the integration between digital and physical interaction. The PEIS Ecology approach is being validated in realistic home environments (see Fig. 6) in order to assess its acceptability for elderly people. Full details about the PEIS Ecology project, including videos, publications, and source code, can be found on the project web site http://aass.oru.se/ peis/.

\section{3) Summary of the contents of the Special Issue}

This Special Issue shows a selection of topics and potential solutions in NRS. Some of the works explain specific solutions for challenging scientific-technological problems, for example robust localisation in outdoor environments; other works propose approaches to deal with task allocation and execution; and finally another work makes a comparative study of Internet protocols fro NRS.

The first two papers discuss some aspects of localisation of robots in a NRS space. The first paper, "A Probabilistic Framework for Entire WSN Localization Using a Mobile Robot”, presents a method for the localization of a Wireless Sensor Network (WSN) by means of collaboration with a robot within a NRS, by employing the signal strength as input. The second paper, "Action Evaluation for Mobile Robot Global Localization in Cooperative Environments", presents a theoretical solution to the global localisation of mobile robots operating in large and cooperative environments. The method is based on estimating the pose of a robot in the map reference frame, then it generates the position hypotheses, and it uses a 
probabilistic active strategy to select the movement of the robot to disambiguate its position. The remote observations available in NRS are used to do this task.

The next couple of papers deal with aspects of cooperation in a NRS. The third paper, "Autonomous Functional Configuration of a Network Robot System", proposes an approach to automatically generate a functional configuration of a NRS to perform, in a cooperative way, a given task in a given environment. This approach also allows a NRS to dynamically change its configuration in response to failures. The fourth paper, "Framework and Service Allocation for Network Robot Platform and Execution of Interdependent Services", presents a framework of three layers (connection units, the area management gateway and the robot-user) to cope with robot service allocation and task execution.

The fifth paper, "Robots in the kitchen: Exploiting ubiquitous sensing and actuation", proposes a NRS that allows a service robot to operate autonomously in a sensor-equipped kitchen. The approach is based on the Player architecture, suitably extended to operate in a NRS setting.

The sixth paper, "Human Behavior Recognition Using Unconscious Cameras and a Visible Robot in a Network Robot System", presents a human behavior recognition method in a NRS scenario, to provide guidance in a public space. They present some experiments showing the recognition of the basic features.

All of the above papers focus on the "autonomy" aspect of a NRS. The seventh and last paper, "End-toEnd Congestion Control Protocols for Remote Programming of Robots using Heterogeneous Networks: A Comparative Analysis", focuses on the other facet of a NRS: tele-operation. The authors propose a comparative study of variable bandwidth and time-delays of Internet protocols on NRS and give recommendations for the design of End-to-End Congestion Control transport protocol for Internet Telerobotics.

\section{5) Acknowledgements}

This work has been partially supported by CICYT project DPI2007-61452, Consolider Ingenio 2010 (CSD2007-00018), IST-045062 of the European Community Union, and the EURON Special Interest Group on Cooperative Robotics (http://www.aass.oru.se/Agora/EuronCoop).

\section{6) References}

[1] A. Sanfeliu and J. Andrade-Cetto, "Ubiquitous networking robotics in urban settings", Workshop on Network Robot Systems. Toward Intelligent Robotic Systems Integrated with Environments. Proceedings of 2006 IEEE/RSJ International Conference on Intelligence Robots and Systems (IROS2006), Beijing, China, Oct. 10-13, 2006.

[2] M. Shiomi, T. Kanda, H. Ishiguro, and N. Hagita, "Interactive Humanoid Robots for a Science Museum”, IEEE Intelligent Systems, vol. 22, no. 2, pp. 25-32, Mar/Apr, 2007.

[3] A. Saffiotti and M. Broxvall, "PEIS Ecologies: Ambient Intelligence meets Autonomous Robotics.", Proc. of the Int. Conf. on Smart Objects and Ambient Intelligence, pp. 275-280. Grenoble, France, 2005. Online at http://aass.oru.se/ peis/. 